\title{
The No-apnea score vs. the other five questionnaires in screening for obstructive sleep apnea-hypopnea syndrome in patients with cerebral infarction
}

\author{
Riken Chen ${ }^{1 \#}$, Wang Liu ${ }^{1 \#}$, Junfen Cheng ${ }^{1 \#}$, Zhenzhen Zheng ${ }^{1 \#}$, Manqing $\mathrm{Ba}^{3}$, Min Peng ${ }^{1}$, Qizhong $\mathrm{He}^{1}$, \\ Huajing Yang ${ }^{2}$, Tao Chen ${ }^{1}$, Cheng Hong ${ }^{2}$
}

${ }^{1}$ Department of Respiratory Medicine, The Second Affiliated Hospital of Guangdong Medical University, Zhanjiang 524023, China; ${ }^{2}$ Department of Respiratory Medicine, State Key Laboratory of Respiratory Disease, the First Affiliated Hospital of Guangzhou Medical University, Guangzhou Institute of Respiratory Health, National Clinical Research Center for Respiratory Disease, Guangzhou 510120, China; ${ }^{3}$ Department of Biochemistry and Chemistry, The University of Arizona, Tucson, AR, USA

Contributions: (I) Conception and design: R Chen, C Hong; (II) Administrative support: J Cheng,W Liu; (III) Provision of study materials or patients: Z Zheng; (IV) Collection and assembly of data: M Ba, M Peng, Q He; (V) Data analysis and interpretation: H Yang, T Chen; (VI) Manuscript writing: All authors; (VII) Final approval of manuscript: All authors.

"These authors contributed equally to this work.

Correspondence to: Cheng Hong. Department of Respiratory Medicine, State Key Laboratory of Respiratory Disease, The First Affiliated Hospital of Guangzhou Medical University, Guangzhou Institute of Respiratory Health, National Clinical Research Center for Respiratory Disease, 151 Yanjiang Road, Guangzhou 510120, Guangdong, China. Email: gyfyyhc@126.com.

Background: Given the growing number of patients suspected of having obstructive sleep apneahypopnea syndrome (OSAHS), screening methods have become increasingly important for sleep clinics. We analyzed the clinical value of the No-apnea score which is used to diagnose OSAHS in patients with cerebral infarction, and compared the accuracy of the No-apnea score with the accuracy of the NoSAS score, the STOP-Bang questionnaire (SBQ), the Epworth Sleepiness Scale (ESS), the STOP questionnaire (STOP) and the Berlin questionnaire (BQ).

Methods: Between January 2014 and December 2018, a total of 221 cerebral infarction patients, suspected of having OSAHS, underwent the polysomnography (PSG) for one night at the sleep medical center of Guangdong Medical University Affiliated Second Hospital. The PSG data were collected and analyzed with the NoSAS score, the SBQ, the ESS, the STOP, the BQ, and patients' demographic information. Based on the apnea-hypopnea index (AHI), the patients were classified into four groups: the normal group ( $<5$ events/h), mild OSAHS group (5-15 events/h), moderate OSAHS group (15-30 events/h) and severe OSAHS group ( $\geq 30$ events/h). The sensitivity, specificity, positive predictive value, negative predictive value and areas under the curve (AUC) of the Receiver Operating Curve (ROC) were calculated for the five questionnaires to compare their relative efficacies for diagnosing OSAHS.

Results: When using the standard of AHI $\geq 5$ for diagnosing OSAHS, the NoSAS score had an AUC of 0.831; the SBQ had an AUC of 0.730; the BQ had an AUC of 0.698; and the STOP had an AUC of 0.735, so these techniques are relatively accurate in diagnosing OSAHS. On the other hand, the No-apnea score and the ESS score are relatively less accurate comparing to the rest: the No-apnea had an AUC of 0.626, and the ESS had an AUC of 0.650. Using the NoSAS score to predict AHI $\geq 5$ events/h, AHI $\geq 15$ events/h and $\mathrm{AHI} \geq 30$ events/h, the sensitivity and specificity were 0.867 and $0.731,0.888$ and $0.476,0.889$ and 0.369 , respectively; Using the SBQ to predict AHI $\geq 5$ events/h, AHI 15 events/h and AHI $\geq 30$ events/h, the sensitivity and specificity were 0.903 and $0.268,0.914$ and $0.200,0.903$ and 0.268 , respectively; Using the STOP to predict $\mathrm{AHI} \geq 5$ events/h, AHI $\geq 15$ events/h and $\mathrm{AHI} \geq 30$ events/h, the values were 0.830 and 0.500, 0.871 and 0.390, 0.875 and 0.302, respectively; and using the BQ to predict $\mathrm{AHI} \geq 5$ events/h, AHI $\geq 15$ events/h and AHI $\geq 30$ events/h, the values were 0.758 and $0.482,0.810$ and $0.429,0.819$ and 0.362 , respectively. 


\begin{abstract}
Conclusions: The study concludes that the NoSAS score and the SBQ had a better predictive value for cerebral infarction patients suspected with OSAHS disease. These questionnaires can also effectively help clinicians quickly address nocturnal hypoxia in patients with cerebral infarction to control subsequent complications in patients with cerebral infarction. More studies are needed to evaluate the efficacy of the NoSAS score in screening for OSAHS in patients with cerebral infarction.
\end{abstract}

Keywords: NoSAS score; obstructive sleep apnea-hypopnea syndrome (OSAHS); cerebral infarction; diagnosing

Submitted Apr 20, 2019. Accepted for publication Sep 26, 2019.

doi: $10.21037 /$ jtd.2019.09.75

View this article at: http://dx.doi.org/10.21037/jtd.2019.09.75

\section{Introduction}

Obstructive sleep apnea-hypopnea syndrome (OSAHS) mainly causes apnea during sleep due to by various factors. OSAHS lead to chronic intermittent anoxia, hypercapnia, and sleep fragmentation, and ultimately culminating in systematic disease (1), including cardiovascular diseases, cerebrovascular diseases, pulmonary vessel diseases and metabolic disease. Epidemiological statistics (2) showed that approximately $2 \%$ of females and $4 \%$ of males are diagnosed with OSAHS, and recent studies found that OSAHS portends a high morbidity. For example, Peppard et al. reported that OSAHS affects approximately $34 \%$ of males and $17 \%$ females to different degrees (3). OSAHS is a common complication in cerebral infarction patients, and the morbidity rate of OSAHS in patients with cerebral infarction is significantly higher than in the general population (4). Specifically, research shows that ischemic patients are 3 to 4 times more likely to be diagnosed with OSAHS compared to the general population (5). In addition, the incidence of hospitalization and the 6-month mortality rate after stroke events were both increased among OSAHS patients (6). After following 132 patients over a period of 10 years, Sahlin et al. reported that OSAHS was an independent risk factors for death among post ccerebral infarction patients. The OSAHS risk factor is independent of age, gender, body mass index (BMI), smoking status, hypertensive diseases, cerebral infarction, atrial fibrillation, cognitive competence, etc. (7). Polysomnography (PSG) examination, the current gold standard in the diagnosis of OSAHS, is not in widespread use, especially in small local hospitals, due to many limitations: PSG is time-consuming, difficult to operate, expensive, and requires a professional examination room and specialized technicians. As a result, convenient yet precise diagnostic tools are needed to identify people with suspected OSAHS. Diagnosis tools such as the Berlin questionnaire (BQ), STOP-Bang questionnaire (SBQ), and STOP questionnaire (STOP) have recently been widely applied in China for the detection of OSAHS. In addition, the Epworth sleepiness scale (ESS), a scale that subjectively evaluates the severity of daytime sleepiness by patients, is regarded as another commonly used detection tool for OSAHS in China. Ricardo recently introduced the No-apnea mnemonic model which only has two variables, age and neck circumference, and reported that the Noapnea model exhibits similar prediction efficiency compared with the NoSAS score, and may also represent a reliable tool for identifying OSAHS (8). The NoSAS score, one of newest screening tools, is similar to the NAMES assessment but easier to remember (9). The NoSAS score was first tested on 2,121 Swiss individuals (HypnoLaus) in a cohort project by Marity-Soler et al. at the University of Lausanne, Switzerland, and the results were relatively accurate. The accuracy of the NoSAS was validated by the Brazil study group involving 1,042 subjects (EPISONO) and other scholars (10). Both research teams proved that the NoSAS score can be used as an efficient and effective detection tool to evaluate the risk of patients suspected of OSAHS. Both studies were based on the local community population, not the clinical cerebral infarction population, and none of correlative research specifically evaluated the predictive value of the NoSAS score in the clinical cerebral infarction population. However, the NoSAS includes overweight and age among its variables, which are also common significant factors in cerebral infarction disease. The No-apnea and NoSAS scores may aid in the identification of OSAHS in cerebral infarction patients. Therefore, this study reviewed and analyzed 221 cerebral infarction patients at the Second affiliated hospital of Guangdong Medical University by simultaneously applying the No-apnea, NoSAS, ESS score, STOP, SBQ, and BQ, statistically analyzed the data 
using PSG, evaluated the efficacy of the NoSAS score in diagnosing cerebral patients, and compared the diagnostic effectiveness of the different methods.

\section{Methods}

\section{Subjects}

Data from 221 cerebral infarction patients who were monitored by overnight PSG at the Institute of Sleep Disease of the Second Affiliated Hospital of the Guangdong Medical University were retrospectively analyzed. Patient inclusion criteria were as follows: patients who were diagnosed with cerebral infarction, patients who were 18 years of age or older, patients who exhibit independent behavioral and cognitive abilities, and patients who are able to complete data about the correlative questionnaire and anthropometric measures. Patient exclusion criteria were as follows: patients on home oxygen therapy or patients with a history of arterial hypertension, active/ unstable cardiovascular diseases, brain tumor, epilepsy, sleep disorders other than OSAHS, neuromuscular disease or other lung diseases that can affect the blood oxygenation, patients taking benzodiazepines, patients undergoing treatment for OSAHS, and patients with incomplete data.

\section{Study methods}

Demographic data from cerebral infarction patients admitted for PSG were collected. Demographic data collection included age, gender, occupation, and education. The data collection also included anthropometric measures, including height, weight, neck circumference, and wrist circumference. In addition, records of the overnight sleep status, such as the severity or time of patients' snoring (S) and apnea were collected. After data collection, various questionnaires were applied and analyzed. The No-apnea score ranges from 0 to 9 points and includes two variables: neck circumference and age. Patients who have a neck circumference less than $37.0 \mathrm{~cm}$ are given 0 points; patients who have neck circumference between 37.0 to $39.9 \mathrm{~cm}$, 1 point; patients who have neck circumference between 40.0 to $42.9 \mathrm{~cm}, 3$ points; and patients who have neck circumference greater than or equal to $43.0 \mathrm{~cm}, 6$ points. Patients less than 35 years old are scored 0 points; patients who are between age 35 to 44 years, 1 point; patients who are between 45 to 54 years, 2 points; patients who are greater than or equal to 55 years, 3 points. If the patient scores higher than 3 points, the patient is identified as having a high risk OSAHS. The NoSAS scores range from 0 to 17 points and include 5 factors: neck circumference $(\mathrm{N})$, BMI, snoring (S), age (A), and gender. Patients with $\mathrm{N}$ greater than $40 \mathrm{~cm}$ score 4 points; patients with BMI between 25 to $30 \mathrm{~kg} / \mathrm{m}^{2}$ score 3 points; patients with BMI greater than or equal to $30 \mathrm{~kg} / \mathrm{m}^{2}$ score 5 points. Patients score 2 points for snoring. Patients who are older than 55 years score 4 points. Male patients score 2 points. Patients with more than 8 points are at higher risk of OSAHS (11). The SBQ was established by adding the Bang to the STOP (including 4 factors, snoring, fatigue apnea and hypertension): B (BMI more than $35 \mathrm{~kg} / \mathrm{m}^{2}$ ), A (age more than 50 years old), $\mathrm{N}$ (neck circumference more than $40 \mathrm{~cm}$ ), and $\mathrm{G}$ (male). If any of the answers to the factors above is yes, then the patient scores 1 point; if the answer is no, then the patient scores 0 points. When the total score is greater than 3 points, the patient is at highrisk for OSAHS (12). The BQ (13) has been widely applied as a diagnostic tool for OSAHS worldwide and includes 11 questions in 3 groups: severity of snoring, daytime sleepiness and hypertension or obesity. If two groups or more are positive, then the person is at high risk of OSAHS. The ESS consists of 8 questions, allowing the subjects to assess their degree of dozing in a particular scene during the day, 0 for no dozing, and 1, 2, and 3 for light, moderate, and severe dozing, patients with more than 8 points are in higher risk of diagnosing OSAHS. After performing different questionnaire, all patients underwent a standard PSG (14), by Alice5 PSG (Philips), requires monitoring the patients for at least $7 \mathrm{~h}$. The participants were asked to refrain from drinking alcohol, coffee, sedatives or hypnotics on the day of the examination. The monitor index includes blood oxygen saturation, electroencephalography (EEG), electromyography (EMG), electrocardiography (ECG), snoring noise, oronasal airflow as evaluated with a thermistor sensor, thoracoabdominal breathing, and posture. According to the interpretation guidelines about sleep and correlative events of 2017 edition of American academy of sleep medicine (AASM) (15). Apnea was defined as a decrease of at least $90 \%$ of airflow from baseline, lasting 10 second or longer. Hypopnea was defined as a decrease of at least $30 \%$ of airflow from baseline, lasting 10 seconds and associated with either an arousal or a $\geq 3 \%$ $\mathrm{O}_{2}$ saturation decrease. The data were interpreted by the professional sleep technicians to determine the sleep stages and the sleep breathing events, then rectified by the same doctor at the end. Both the diagnosis and severity 
Table 1 The general data of 221 cerebral infraction patients

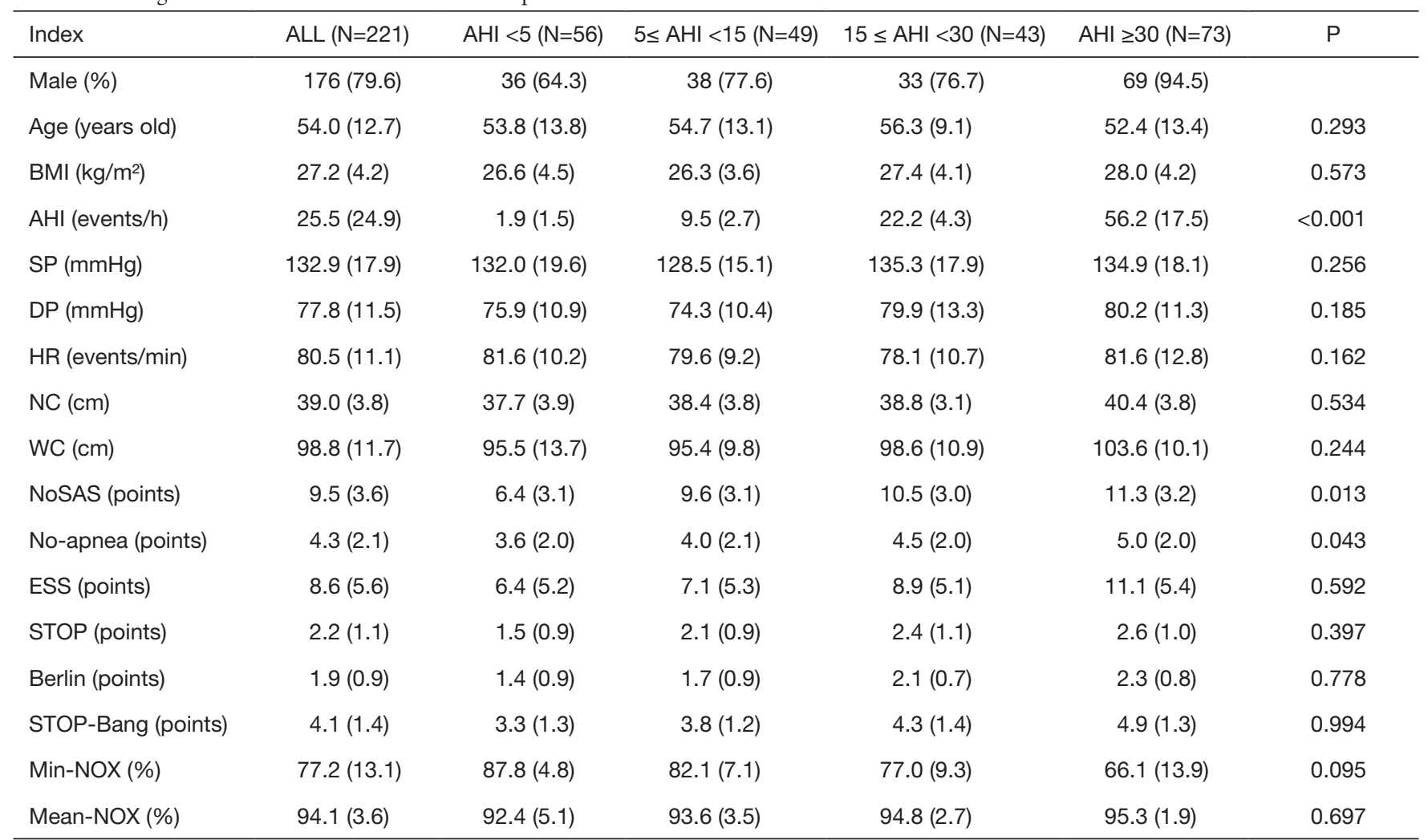

BMI, body mass index; AHI, apnea hypopnea index; SP, systolic pressure; DP, diastolic pressure; HR, heart rate; NC, neck circumference; WC, wrist circumference; ESS, Epworth Sleepiness Scale; Min-NOX, minimum nocturnal oxygen saturation; Mean-NOX, mean nocturnal oxygen saturation; $\mathrm{P}, \mathrm{P}$ value.

of OSAHS were classified based upon the AHI: $>5-15 / \mathrm{h}$ mild, $>15-30 / \mathrm{h}$ moderate, and $>30 / \mathrm{h}$ severe (15), OSAHS was diagnosed when the apnea-hypopnea index (AHI) was greater than or equal to 5 times/h. In addition, the grade of illness was used to classified the patients into a snore group (AHI $<5$ times/h), the mild OSAHS group (AHI 5 to 15 times/h), the moderate OSAHS group (AHI 15 to 30 times/h) and the severe OSAHS group (AHI $\geq 30$ times/h). Additional diagnoses obtained throughout the sleep study were defined accordingly to the International Classification of Sleep disorders-third edition (16). The study was approved by the Institutional Review Board (IRB number: 2018091) and written informed consent was obtained from all patients.

\section{Statistical analysis}

All data were analyzed by SPSS 16.0 statistical software. The data for normally distributed data were calculated as the mean \pm standard deviation, and one-way ANOVA was used for mean value comparison. A post hoc analysis was used to compare the two groups. Count data were expressed as rate, and the $\mathrm{x}^{2}$ test was used for comparisons between groups. $\mathrm{P}<0.05$ was defined as statistically significant. The sensitivity, specificity, positive predictive value and negative predictive value of each scale were calculated in a four-grid table. The diagnostic efficacy of each scale to diagnose OSAHS was analyzed and evaluated by operating characteristic curves.

\section{Results}

Finally, we collected 221 patients with cerebral infarction and 51 were excluded according to the exclusion criteria. It had $71.1 \%, 85.5 \%, 79.8 \%, 69.7 \%, 74.7 \%$ and $86.0 \%$ of individuals classified as high-risk for NoSAS, Noapnea, ESS, Berlin, STOP and STOP-Bang, respectively. General data (Table 1): among 221 cerebral infarction patients with suspected OSAHS, 176 (79.6\%) were male. The mean patient age was $54.0 \pm 12.7$ years old; the mean 


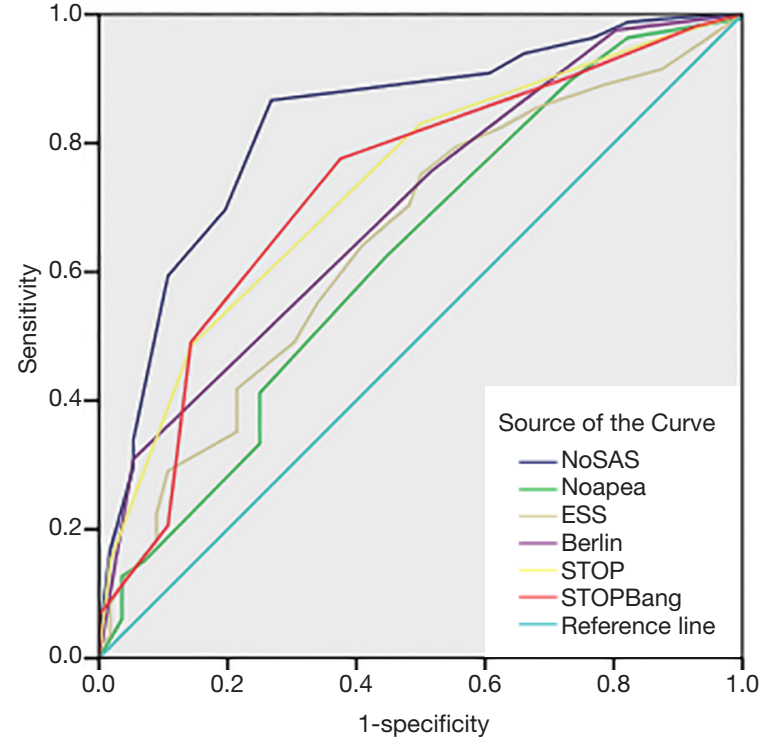

Figure 1 ROC curve of the six screening tools at AHI cutoff of $\geq 5$ events/h. ROC, receiver operating curve; AHI, apneahypopnea index; ESS, Epworth Sleepiness Scale; STOP, STOP questionnaire.

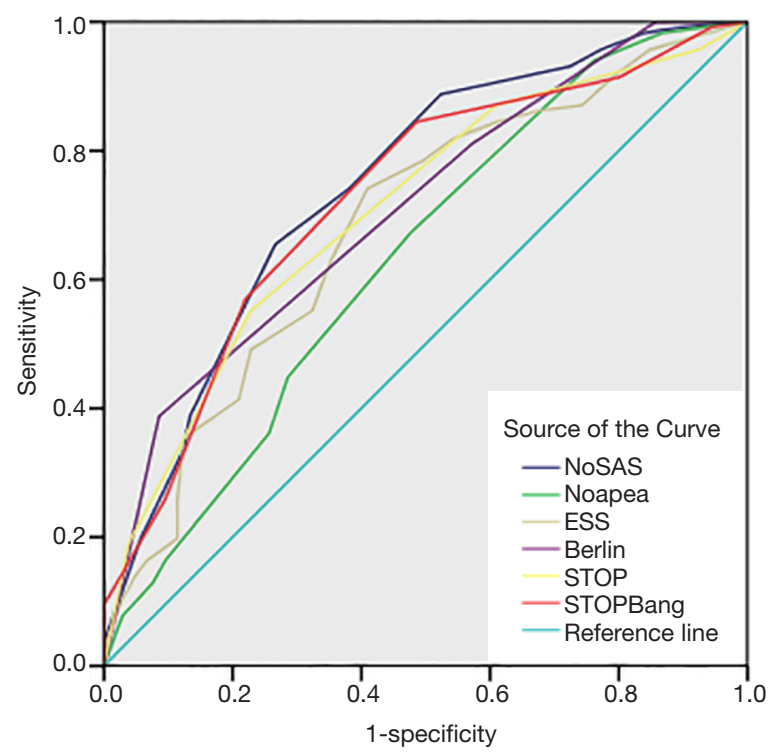

Figure 2 ROC curve of the six screening tools at AHI cutoff of $\geq 15$ events/h. ROC, receiver operating curve; AHI, apneahypopnea index; ESS, Epworth Sleepiness Scale; STOP, STOP questionnaire.

BMI was $27.2 \pm 4.2 \mathrm{~kg} / \mathrm{m}^{2}$; the mean neck circumference was $39.0 \pm 3.8 \mathrm{~cm}$; and the mean waist circumference was $98.8 \pm 11.7 \mathrm{~cm}$. The mean scores of the NoSAS, SBQ, ESS,

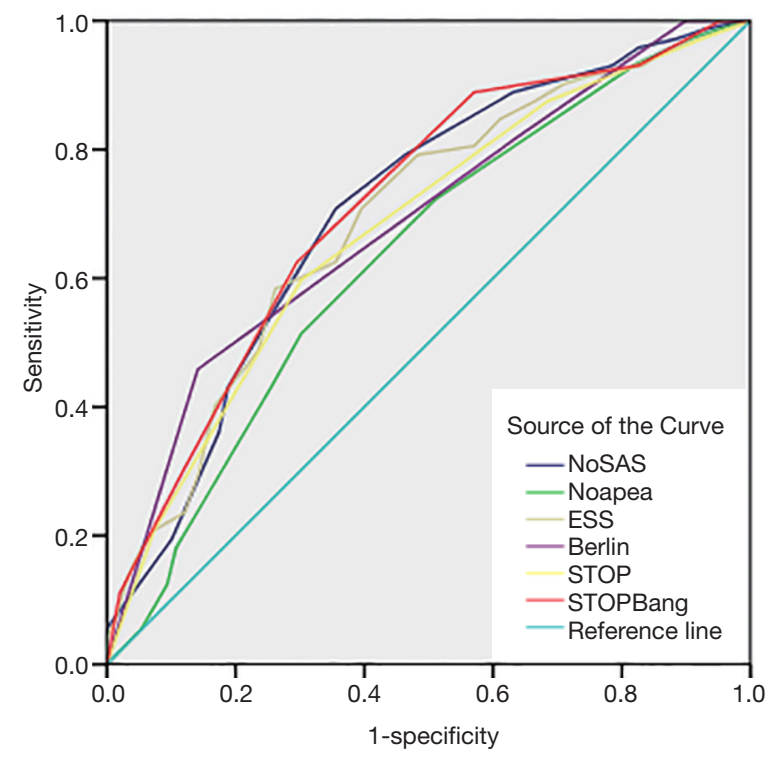

Figure 3 ROC curve of the five screening tools at AHI cutoff of $\geq 30$ events/h. ROC, receiver operating curve; AHI, apneahypopnea index; ESS, Epworth Sleepiness Scale; STOP, STOP questionnaire.

STOP, and Berlin tests were 9.5 $\pm 3.6,4.1 \pm 1.4,8.6 \pm 5.6$, $2.2 \pm 1.1$, and $1.9 \pm 0.9$, respectively. There were $56(25.3 \%)$ cases in the pure snoring group, $49(22.2 \%)$ cases in the mild OSAHS group, $43(19.5 \%)$ cased in the moderate OSAHS group, and $73(33.0 \%)$ cases in the severe OSAHS group. There were statistically significant differences $(\mathrm{P}<0.001)$ in the NoSAS scores between the four groups, but there were not statistically significant differences (all $\mathrm{P}>0.05$ ) in the ESS scores, STOP, STOP-Bang and BQs among the groups. However, we identified an increasing trend in the scores of those questionnaires when the AHI was increased.

The screening effectiveness of each scale (Figures 1-3): With the cutoff value of AHI 5, 15 and 30 times/hour, the research team compared the area under the curve (AUC) and generated ROC curves for the four scales, including the No-apnea, NoSAS score, SBQ, ESS score, STOP and $B Q$. The results revealed that the NoSAS had the largest AUC at the cutoff point of AHI $\geq 5,15 / \mathrm{h}$. At the cutoff point of AHI $\geq 5 / \mathrm{h}$, the AUCs of NoSAS obtained was better than others $(\mathrm{P}<0.05)$. At the cutoff point of $\mathrm{AHI} \geq 15$, $30 / \mathrm{h}$, the differences of the AUCs found between the various instruments had no significance $(\mathrm{P}>0.05)$. STOPBang had the largest AUC at the cutoff point of $\mathrm{AHI} \geq 30$.

The sensitivity and specificity of the NoSAS scores 
Table 2 With cut-off point of AHI of 5 as the diagnose of OSAHS disease

\begin{tabular}{lcclll}
\hline Questionnaire & AUC $(95 \% \mathrm{Cl})$ & Sensitivity $(95 \% \mathrm{Cl})$ & Specificity $(95 \% \mathrm{Cl})$ & PPV $(95 \% \mathrm{Cl})$ & $\mathrm{NPV}(95 \% \mathrm{Cl})$ \\
\hline NoSAS & $0.831(0.768-0.894)$ & $0.867(0.815-0.919)$ & $0.732(0.537-0.785)$ & $0.905(0.859-0.951)$ & $0.651(0.833-0.769)$ \\
No-apnea & $0.626(0.539-0.714)$ & $0.897(0.851-0.943)$ & $0.268(0.152-0.384)$ & $0.783(0.724-0.842)$ & $0.469(0.296-0.642)$ \\
ESS & $0.650(0.568-0.775)$ & $0.552(0.476-0.627)$ & $0.661(0.351-0.613)$ & $0.827(0.759-0.898)$ & $0.333(0.246-0.421)$ \\
Berlin & $0.698(0.620-0.808)$ & $0.758(0.692-0.823)$ & $0.482(0.369-0.631)$ & $0.812(0.750-0.873)$ & $0.403(0.286-0.520)$ \\
STOP & $0.735(0.662-0.808)$ & $0.830(0.773-0.888)$ & $0.500(0.369-0.631)$ & $0.830(0.773-0.888)$ & $0.500(0.369-0.631)$ \\
STOP-Bang & $0.730(0.652-0.807)$ & $0.903(0.858-0.948)$ & $0.268(0.152-0.384)$ & $0.784(0.726-0.842)$ & $0.484(0.308-0.660)$ \\
\hline
\end{tabular}

$\mathrm{AHI}$, apnea hypopnea index; OSAHS, obstructive sleep apnea-hypopnea syndrome; AUC, the area under the ROC curve; Cl, confident interval; PPV, positive predictive value; NPV, negative predictive value; ESS, Epworth Sleepiness Scale; STOP, STOP questionnaire.

Table 3 With cut-off point of AHI of 15 as the diagnose of OSAHS disease

\begin{tabular}{lccccc}
\hline Questionnaire & AUC $(95 \% \mathrm{Cl})$ & Sensitivity $(95 \% \mathrm{Cl})$ & Specificity $(95 \% \mathrm{Cl})$ & PPV $(95 \% \mathrm{Cl})$ & $\mathrm{NPV}(95 \% \mathrm{Cl})$ \\
\hline NoSAS & $0.746(0.681-0.811)$ & $0.888(0.831-0.945)$ & $0.476(0.381-0.571)$ & $0.652(0.558-0.726)$ & $0.794(0.694-0.894)$ \\
No-apnea & $0.633(0.560-0.706)$ & $0.930(0.872-0.989)$ & $0.238(0.157-0.320)$ & $0.354(0.286-0.423)$ & $0.844(0.718-0.970)$ \\
ESS & $0.685(0.614-0.755)$ & $0.629(0.541-0.717)$ & $0.648(0.556-0.738)$ & $0.664(0.575-0.752)$ & $0.613(0.522-0.703)$ \\
Berlin & $0.709(0.654-0.776)$ & $0.810(0.739-0.882)$ & $0.429(0.334-0.523)$ & $0.610(0.533-0687)$ & $0.672(0.559-0.784)$ \\
STOP & $0.707(0.639-0.775)$ & $0.871(0.739-0.882)$ & $0.390(0.297-0.484)$ & $0.612(0.538-0.686)$ & $0.732(0.616-0.848)$ \\
STOP-Bang & $0.726(0.659-0.793)$ & $0.914(0.862-0.965)$ & $0.200(0.123-0.277)$ & $0.558(0.487-0.629)$ & $0.677(0.513-0.842)$ \\
\hline
\end{tabular}

$\mathrm{AHI}$, apnea hypopnea index; OSAHS, obstructive sleep apnea-hypopnea syndrome; AUC, the area under the ROC curve; Cl, confident interval; PPV, positive predictive value; NPV, negative predictive value; ESS, Epworth Sleepiness Scale; STOP, STOP questionnaire.

were 0.867 and $0.731,0.888$ and $0.476,0.889$ and 0.369 for AHI $\geq 5$ times/h, AHI $\geq 15$ times/h, and AHI $\geq 30$ times/ $\mathrm{h}$, respectively; the sensitivity and specificity of the Noapnea scores were 0.897 and $0.268,0.931$ and $0.238,0.940$ and 0.181 , for $\mathrm{AHI} \geq 5$ times/h, $\mathrm{AHI} \geq 15$ times/h, and $\mathrm{AHI}$ $\geq 30$ times/h, respectively; the sensitivity and specificity of the SBQ were 0.903 and $0.268,0.914$ and $0.200,0.903$ and 0.268 , for $\mathrm{AHI} \geq 5$ times/h, AHI $\geq 15$ times/h, and AHI $\geq 30$ times/h, respectively; the sensitivity and specificity of the STOP were 0.830 and $0.500,0.871$ and $0.390,0.875$ and 0.302 , respectively; and the sensitivity and specificity of the BQs were 0.758 and $0.482,0.810$ and $0.429,0.819$ and 0.362 , respectively (Tables 2-4).

\section{Discussion}

OSAHS is a systemic disease that involves many systems of the human body. OSAHS causes intermittent hypoxia, oxidative stress, and inflammation, and can have make significant negative impacts on the human body. OSAHS is a risk factor for cerebral infarction, and treatment of
OSAHS may reduce new first-time cerebrovascular events and recurrences (17). PSG examination, the current gold standard in the diagnosis of OSAHS, is not used widely, especially in small local hospitals, due to its many limitations; PSG is time-consuming, difficult to operate, expensive, and requires a professional examination room and specialized technicians. Therefore, some accessible and accurate methods are urgently needed to diagnose patients suspected of having OSAHS, especially among cerebral infarction patients, because OSAHS can significantly affect the development and complications of the disease. Currently, there are a variety of predictive scales for OSAHS diagnosis, including the Epworth scale, BQ, SBQ, ASA checklist, and sleep apnea questionnaire (18). The SBQ's name abbreviates the content of the questionnaire which allows medical workers to memorize the name easily. The questionnaire can not only predict the risk of patients' with OSAHS disease but also determine the severity of the disease. At the same time, the questionnaire has gradually been recognized and promoted due to its accessibility and simple question content, which is accepted easily by the 
Table 4 With cut-off point of AHI of 30 as the diagnose of OSAHS disease

\begin{tabular}{lcllll}
\hline Questionnaire & AUC $(95 \% \mathrm{Cl})$ & Sensitivity $(95 \% \mathrm{Cl})$ & Specificity $(95 \% \mathrm{Cl})$ & PPV $(95 \% \mathrm{Cl})$ & $\mathrm{NPV}(95 \% \mathrm{Cl})$ \\
\hline NoSAS & $0.705(0.637-0.776)$ & $0.889(0.816-0.961)$ & $0.369(0.292-0.447)$ & $0.405(0.329-0.482)$ & $0.873(0.791-0.955)$ \\
No-apnea & $0.634(0.558-0.710)$ & $0.940(0.896-0.983)$ & $0.181(0.119-0.243)$ & $0.577(0.506-0.647)$ & $0.781(0.638-0.924)$ \\
ESS & $0.693(0.619-0.767)$ & $0.708(0.603-0.813)$ & $0.604(0.526-0.683)$ & $0.464(0.370-0.557)$ & $0.811(0.738-0.884)$ \\
Berlin & $0.688(0.613-0.764)$ & $0.819(0.731-0.908)$ & $0.362(0.285-0.440)$ & $0.383(0.306-0.460)$ & $0.806(0.711-0.901)$ \\
STOP & $0.677(0.602-0.752)$ & $0.875(0.799-0.951)$ & $0.302(0.228-0.376)$ & $0.377(0.304-0.451)$ & $0.833(0.734-0.932)$ \\
STOP-Bang & $0.714(0.642-0.785)$ & $0.931(0.872-0.989)$ & $0.175(0.114-0.235)$ & $0.353(0.285-0.421)$ & $0.839(0.709-0.968)$ \\
\hline
\end{tabular}

$\mathrm{AHI}$, apnea hypopnea index; OSAHS, obstructive sleep apnea-hypopnea syndrome; AUC, the area under the ROC curve; Cl, confident interval; PPV, positive predictive value; NPV, negative predictive value; ESS, Epworth Sleepiness Scale; STOP, STOP questionnaire.

patients. Abrishami (19) demonstrated that SBQ had great predictive efficiency and was easy to use. However, the study was performed in the general population without cerebral infarction; therefore, the SBQ content exhibits low sensitivity for diagnosing cerebral infarction. On the other hand, common characteristics among patients with cerebral infarction or OSAHS, including obesity and large neck circumference, are included in both the NoSAS score and the SBQ. In this study, the research team determined that although the SBQ questionnaire exhibits higher methodology validity and is easier to apply, the average BMI values in all SDB groups were lower than the value limit of the SBQ, which was $35 \mathrm{~kg} / \mathrm{m}^{2}$. Due to the adjustment of BMI in the NoSAS score system, this study determined that the NoSAS exhibits better diagnostic value for OSAHS in cerebral patients. Unlike the NoSAS, the No-apnea score emphasizes the neck circumference and the age of the patients. Associating different scores with different ranges of neck circumference and age has some scientific value, but directly assigning 3 points to patients older than 55 years old, thereby automatically including them in the high-risk OSAHS group, reduces the efficiency. As a result, this study demonstrates that the AUC of the No-apnea is less than 0.7, which is not as efficient as the NoSAS.

This study determined that 165 of $221(74.7 \%)$ cerebral infarction patients were diagnosed with OSAHS disease which indicates that OSAHS is commonly diagnosed in cerebral infarction patients. However, this result might include some selection bias only cerebral infarction patients suspected of having OSAHS disease were recruited for PSG. This study also determined that the proportion of the male OSAHS patients (140/176, 79.5\%) was much higher than the proportion of female patients $(25 / 45,55.6 \%)$, and this result is consistent with the results reported in the literature (20). The ideal screening scale should have both a large AUC (21) and high sensitivity to avoid false negative results. In this study, AHI $\geq 5$ was used as the diagnostic criterion for OSAHS. The AUC of the NoSAS score was 0.831 which exceeds 0.7 , and indicates that the NoSAS possesses good predictive value. In addition, the AUC values of the STOP, No-apnea score and SBQ were $0.735,0.730$ and 0.730 , respectively, which were also above 0.7 ; thus, these methods also have good predictive values. On the other hand, the AUC values of the BQ and ESS score were 0.698 and 0.650 , respectively which means that they have slightly lower predictive values compared with the methods mentioned before. In addition, this study confirmed that the NoSAS score exhibits good discrimination value for identifying OSAHS patients by using the ROC curve to calculate the AUC. The sensitivities of all questionnaires were compared. When taking the cut-off of 5 times/h as the OSAHS diagnostic standard, the NoSAS score, Noapnea score, SBQ, STOP and BQ exhibited sensitivities of $0.867,0.903,0.903,0.830$ and 0.758 , respectively. When taking AHI $\geq 15$ times/h as the OSAHS diagnostic standard, the sensitivities of the No-apnea score, NoSAS score, SBQs, STOPs and BQs were 0.930, 0.888, 0.914, 0.871 and 0.810 , respectively. Using the cut-off of 30 as the OSAHS diagnostic standard, the sensitivities of the Noapnea score, NoSAS score, SBQs, STOPs and BQs were $0.940,0.889,0.931,0.875$ and 0.819 , respectively. When it came to the cutoff point of AHI of 5,15 times/h, the sensitivity of the No-apnea score was the highest among all diagnostic questionnaires, followed by the SBQ whose specificity was lower than the NoSAS. The study by Chung et al used the SBQ to predict the degree of obesity, and the SBQ's sensitivity to diagnose OSAHS disease was $90.5 \%$ with a specificity of $28.1 \%$. However, in this study, when comparing the different cutoff points of AHI of 5, 15 and 30 , the sensitivity of the SBQ was higher but the specificity 
was lower. Furthermore, this study demonstrates that the SBQ exhibits high accuracy due to the relatively large AUC. Although NoSAS exhibits slightly lower sensitivity, its specificity is significantly higher. Thus, the NoSAS exhibits higher adaptability among the Chinese cerebral infarction population compared with other questionnaires, likes other studies (22-24), so it deserved to be applied widely among cerebral infarction patients who are suspected of having OSAHS. The subsequent treatment of OSAHS can then be applied to reduce new first-time cerebrovascular events and recurrences.

The advantage of this study is that it represents one of the latest studies in the clinical study to analyze the applied value of the No-apnea score, NoSAS score and other four questionnaires in diagnosing cerebral infarction patients with the suspected of OSAHS. Also, No-apnea, NoSAS score and SBQ were targeted towards the main characteristics, obesity and large neck circumference, in cerebra infarction patients. The subjects in our study were local urban local residents which suggests the sample has the great representative characteristic.

\section{Limitations}

This study is a retrospective single-center study with a relatively small sample consisted mainly of obese men with an average age of over 50 years, it may have conditioned our results .More studies are needed to evaluate the efficacy of this score in younger populations, with a predominance of female and non-obese individuals with the cerebral infarction.

\section{Conclusions}

The study concludes that the NoSAS score and the SBQ had a better predictive value for cerebral infarction patients suspected with OSAHS disease. These questionnaires can also effectively help clinicians quickly address nocturnal hypoxia in patients with cerebral infarction to control subsequent complications in patients with cerebral infarction. More studies are needed to evaluate the efficacy of the NoSAS score in screening for OSAHS in patients with cerebral infarction.

\section{Acknowledgments}

We thank Lishu Zhang and Tuxuan Liu (both the Second Affiliated Hospital of Guangdong Medical University,
Zhanjiang 524023, China) for providing the questionnaire and the data.

Funding: This study was funded by Natural Science Foundation of Guangdong Province (2014A030310219).

\section{Footnote}

Conflicts of Interest: The authors have no conflicts of interest to declare.

Ethical Statement: The authors are accountable for all aspects of the work in ensuring that questions related to the accuracy or integrity of any part of the work are appropriately investigated and resolved. The study was approved by the Institutional Review Board (IRB number: 2018091) and written informed consent was obtained from all patients.

\section{References}

1. Zamarron C, Garcia Paz V, Riveiro A, et al. Obstructive sleep apnea syndrome is a systemic disease. Current evidence. Eur J Intern Med 2008;19:390-8.

2. Coughlin SR, Mawdsley L, Mugarza JA, et al. Obstructive sleep apnoea is independently associated with an increased prevalence of metabolic syndrome. Eur Heart J 2004;25:735-41.

3. Peppard PE, Young T, Barnet JH, et al. Increased prevalence of sleep-disordered breathing in adults. Am J Epidemiol 2013;177:1006-14.

4. Lyons OD, Ryan CM. Obstructive sleep apnea and stroke: hand in hand? J Thorac Dis 2018;10:S1099-101.

5. Dempsey JA, Veasey SC, Morgan BJ, et al. Pathophysiology of sleep apnea. Physiol Rev 2010;90:47-112.

6. Turkington PM, Allgar V, Bamford J, et al. Effect of upper airway obstruction in acute stroke on functional outcome at 6 months. Thorax 2004;59:367-71.

7. Sahlin C, Sandberg O, Gustafson Y, et al. Obstructive sleep apnea is a risk factor for death in patients with stroke: a 10-year follow-up. Arch Intern Med 2008;168:297-301.

8. Duarte RLM, Rabahi MF, Magalhães-da-Silveira FJ, et al. Simplifying the Screening of Obstructive Sleep Apnea With a 2-Item Model, No-Apnea: A Cross-Sectional Study. J Clin Sleep Med 2018;14:1097-107.

9. Subramanian S, Hesselbacher SE, Aguilar R, et al. The NAMES assessment: a novel combined-modality screening tool for obstructive sleep apnea. Sleep Breath 
2011;15:819-26.

10. Coutinho Costa J, Rebelo-Marques A, Machado JN, et al. Validation of NoSAS (Neck, Obesity, Snoring, Age, Sex) score as a screening tool for obstructive sleep apnea: Analysis in a sleep clinic. Pulmonology 2019; 25:263-70.

11. Marti-Soler H, Hirotsu C, Marques-Vidal P, et al. The NoSAS score for screening of sleep-disordered breathing: a derivation and validation study. Lancet Respir Med 2016;4:742-8.

12. Chung F, Yegneswaran B, Liao P, et al. STOP questionnaire: a tool to screen patients for obstructive sleep apnea. Anesthesiology 2008;108:812-21.

13. Netzer NC, Stoohs RA, Netzer CM, et al. Using the Berlin Questionnaire to identify patients at risk for the sleep apnea syndrome. Ann Intern Med 1999;131:485-91.

14. Johns MW. A new method for measuring daytime sleepiness: the Epworth Sleepiness Scale. Sleep 1991;14:540-5.

15. Berry R, Brooks R, Gamaldo C, et al. AASM Scoring Manual Updates for 2017 (Version 2.4). J Clin Sleep Med 2017;13:665-6.

16. Sateia MJ. International classification of sleep disordersthird edition: highlights and modifications. Chest 2014;146:1387-94.

17. Cho ER, Kim H, Seo HS, et al. Obstructive sleep apnea as a risk factor for silent cerebral infarction. J Sleep Res 2013;22:452-8.

Cite this article as: Chen R, Liu W, Cheng J, Zheng Z, Ba M, Peng M, He Q, Yang H, Chen T, Hong C. The No-apnea score $v s$. the other five questionnaires in screening for obstructive sleep apnea-hypopnea syndrome in patients with cerebral infarction. J Thorac Dis 2019;11(10):4179-4187. doi: 10.21037/ jtd.2019.09.75
18. Ramachandran SK, Josephs LA. A meta-analysis of clinical screening tests for obstructive sleep apnea. Anesthesiology 2009;110:928-39.

19. Abrishami A, Khajehdehi A, Chung F. A systematic review of screening questionnaires for obstructive sleep apnea. Can J Anesth 2010;57:423-38.

20. Ip MS, Lam B, Tang LC, et al. A community study of sleep-disordered breathing in middle-aged Chinese women in Hong Kong: prevalence and gender differences. Chest 2004;125:127-34.

21. Silva GE, Vana KD, Goodwin JL, et a1. Identification of patients with sleep disordered breathing: comparing the four-variable screening tool, STOP, STOP-Bang, and Epworth Sleepiness Scales. J Clin Sleep Med 2011;7:467-72.

22. Tan A, Hong Y, Tan LWL, et al. Validation of NoSAS score for screening of sleep-disordered breathing in a multiethnic Asian population. Sleep Breath 2017;21:1033-8.

23. Guichard K, Marti-Soler H, Micoulaud-Franchi JA, et al. The NoSAS score: A new and simple screening tool for obstructive sleep apnea syndrome in depressive disorder. J Affect Disord 2018;227:136-40.

24. Cicero AFG, Rosticci M, Pengo MF, et al. NoSAS score associated with arterial stiffness in a large cohort of healthy individuals. Lancet Respir Med 2016;4:e54. 